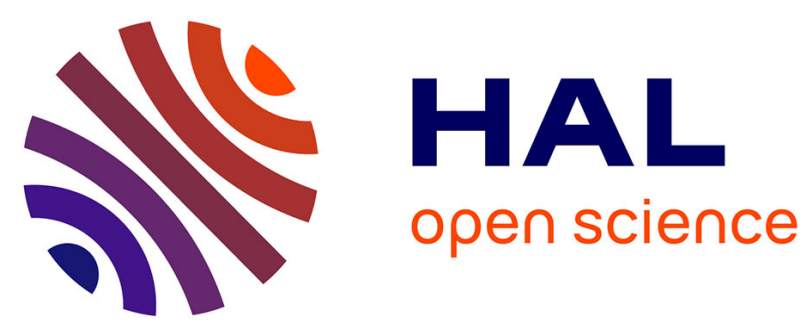

\title{
Femtosecond laser excitation dynamics of the semiconductor-metal phase transition in VO2
}

Michael F. Becker, A. Bruce Buckman, Rodger M. Walser, Thierry Lepine, Patrick Georges, Alain Brun

\section{- To cite this version:}

Michael F. Becker, A. Bruce Buckman, Rodger M. Walser, Thierry Lepine, Patrick Georges, et al.. Femtosecond laser excitation dynamics of the semiconductor-metal phase transition in VO2. Journal of Applied Physics, 1996, 79 (5), pp.2404-2408. 10.1063/1.361167 . hal-00761582

HAL Id: hal-00761582

https://hal-iogs.archives-ouvertes.fr/hal-00761582

Submitted on 15 Apr 2016

HAL is a multi-disciplinary open access archive for the deposit and dissemination of scientific research documents, whether they are published or not. The documents may come from teaching and research institutions in France or abroad, or from public or private research centers.
L'archive ouverte pluridisciplinaire HAL, est destinée au dépôt et à la diffusion de documents scientifiques de niveau recherche, publiés ou non, émanant des établissements d'enseignement et de recherche français ou étrangers, des laboratoires publics ou privés. 


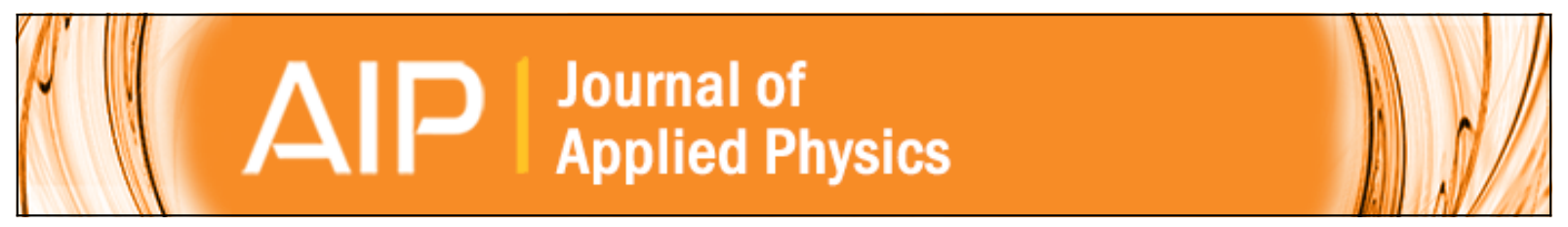

Femtosecond laser excitation dynamics of the semiconductor-metal phase transition in VO2

Michael F. Becker, A. Bruce Buckman, Rodger M. Walser, Thierry Lépine, Patrick Georges, and Alain Brun

Citation: Journal of Applied Physics 79, 2404 (1996); doi: 10.1063/1.361167

View online: http://dx.doi.org/10.1063/1.361167

View Table of Contents: http://scitation.aip.org/content/aip/journal/jap/79/5?ver=pdfcov

Published by the AIP Publishing

\section{Articles you may be interested in}

Optimization of the semiconductor-metal transition in VO2 epitaxial thin films as a function of oxygen growth pressure

Appl. Phys. Lett. 104, 081913 (2014); 10.1063/1.4866806

Anomalous optical switching and thermal hysteresis during semiconductor-metal phase transition of VO2 films on Si substrate

Appl. Phys. Lett. 103, 231114 (2013); 10.1063/1.4838395

Highly tunable-emittance radiator based on semiconductor-metal transition of VO2 thin films

Appl. Phys. Lett. 102, 061107 (2013); 10.1063/1.4792277

Femtosecond laser excitation of the semiconductor-metal phase transition in VO2

Appl. Phys. Lett. 65, 1507 (1994); 10.1063/1.112974

Fast laser kinetic studies of the semiconductor-metal phase transition in VO2 thin films

AIP Conf. Proc. 50, 117 (1979); 10.1063/1.31650

\section{AIP $\left.\right|_{\text {APL Photonics }}$}

APL Photonics is pleased to announce Benjamin Eggleton as its Editor-in-Chief

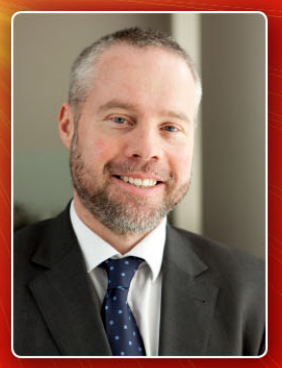




\title{
Femtosecond laser excitation dynamics of the semiconductor-metal phase transition in $\mathrm{VO}_{2}$
}

\author{
Michael F. Becker, ${ }^{\text {a) }}$ A. Bruce Buckman, and Rodger M. Walser \\ Laboratory for Smart Materials and Devices, Department of Electrical and Computer Engineering, \\ The University of Texas at Austin, Austin, Texas 78712 \\ Thierry Lépine, Patrick Georges, and Alain Brun \\ Institut d'Optique Théorique et Appliquée, C.N.R.S., URA No. 14, Université Paris-Sud, B.P. 147, \\ 91403 Orsay, France
}

(Received 21 August 1995; accepted for publication 15 November 1995)

\begin{abstract}
We have measured the subpicosecond optical response of a solid-state, semiconductor-to-metal phase transition excited by femtosecond laser pulses. We have determined the dynamic response of the complex refractive index of polycrystalline $\mathrm{VO}_{2}$ thin-films by making pump-probe optical transmission and reflection measurements at $780 \mathrm{~nm}$. The phase transition was found to be largely prompt with the optical properties very close to the high-temperature metallic state being attained within about 5 ps. The equilibration of the metallic state after femtosecond excitation was modeled by non-exponentially decaying perturbations in the metallic state electron density and collision frequency. The decay of both these plasma parameters was well fit by a $1 / \sqrt{t}$ time dependence. This indicated that a diffusion process governed the equilibration of the metallic phase of $\mathrm{VO}_{2}$. (C) 1996 American Institute of Physics. [S0021-8979(96)06604-2]
\end{abstract}

\section{INTRODUCTION}

The dynamics of solid-state phase transitions are amenable to study by ultrashort pulsed lasers. Ultrafast optical techniques effectively probe the cooperative structural change in the time domain in order to study precursors, soft modes, metastable states, and posttransition relaxations. We have employed femtosecond laser pump-probe techniques to investigate the dynamics of the solid-state phase transition in $\mathrm{VO}_{2}$ near $68^{\circ} \mathrm{C}$. This phase transition incorporates both a lattice-type (symmetry) change and an electronic band change (semiconductor to metal). Femtosecond laser pulses have previously been used to probe the dynamics of solidstate phase transitions in the ferroelectric materials $\mathrm{PbTiO}_{3},{ }^{1}$ $\mathrm{KNbO}_{3}$, and $\mathrm{BaTiO}_{3} .{ }^{2}$ In that case, there was a lattice symmetry change, but the transition was from a nonconducting ferroelectric low-temperature phase to a similarly nonconducting paraelectric phase. In another study of solids, the purely electronic Mott transition in $\mathrm{CuCl}$ had been investigated using femtosecond laser pulses. ${ }^{3}$

The first attempt to measure the switching speed of the optically induced phase transition in $\mathrm{VO}_{2}$ was made by Roach and Balberg ${ }^{4}$ exciting with 20 ns ruby laser pulses at $694 \mathrm{~nm}$ and probing with a cw HeNe laser. They found the transition to be faster than their experimental resolution. Subsequently, Becker ${ }^{5}$ and Walser ${ }^{6}$ conducted the first pumpprobe measurements on the $\mathrm{VO}_{2}$ phase transition using $30 \mathrm{ps}$ pulses from a mode-locked Nd:YAG laser, exciting and probing at $1064 \mathrm{~nm}$. Again, the transition was abrupt and proved to be faster than the experimental resolution. Other experiments in which $\mathrm{VO}_{2}$ films were excited by picosecond pulses at 532 and $1064 \mathrm{~nm}$ did not always show an abrupt transition to the metallic state. ${ }^{7,8}$ In some cases an intermediate state was observed. However, in all the cases where an abrupt

${ }^{\text {a)} E l e c t r o n i c ~ m a i l: ~ b e c k e r @ u t s . u t e x a s . e d u ~}$ transition was observed, the speed was faster than the experimental resolution dictated by $25-30$ ps pulses. The important measurement of the switching time for the phase transition had not yet been made and was an important motivation for our work.

In the experiments reported here, we have measured the subpicosecond response of the optical parameters of $\mathrm{VO}_{2}$ thin films for laser excitation through the first-order phase transition. Femtosecond laser excitation at $780 \mathrm{~nm}$ from an amplified, self-mode-locked Ti:sapphire laser was used in a pump-probe configuration to excite the $\mathrm{VO}_{2}$ film through the phase transition, starting from the low-temperature equilibrium state, a semiconductor with a monoclinic unit cell, to the high-temperature state, a metal with a rutile unit cell. The transient optical reflection and transmission were measured on a femtosecond and picosecond time scale by a delayed probe pulse at $780 \mathrm{~nm}$. The slower behavior for nanosecond and longer times was measured using a cw HeNe laser at 633 $\mathrm{nm}$ and a fast photodiode $\left(\tau_{\text {rise }} \approx 1 \mathrm{~ns}\right)$. We have transformed the raw measurements of dynamic reflection and transmission to complex refractive index using a thin-film model. In a previous letter, ${ }^{9}$ we reported picosecond time scale equilibration effects after the femtosecond pulsed laser excitation of the phase transition. We suggested that perhaps this might indicate deviation of the metallic plasma from the equilibrium conditions described by Barker et al. ${ }^{10}$ and Berglund and Guggenheim ${ }^{11}$ for metallic $\mathrm{VO}_{2}$. In this article, we follow this suggestion by modeling the transient behavior of the laser-excited phase transition.

\section{EXPERIMENTAL METHODS}

The $\mathrm{VO}_{2}$ samples used in these experiments were fabricated by a new low-temperature process compatible with polymer substrates. ${ }^{12}$ In summary, the process involved sputtering vanadium in an oxygen-hydrogen-argon plasma at a 


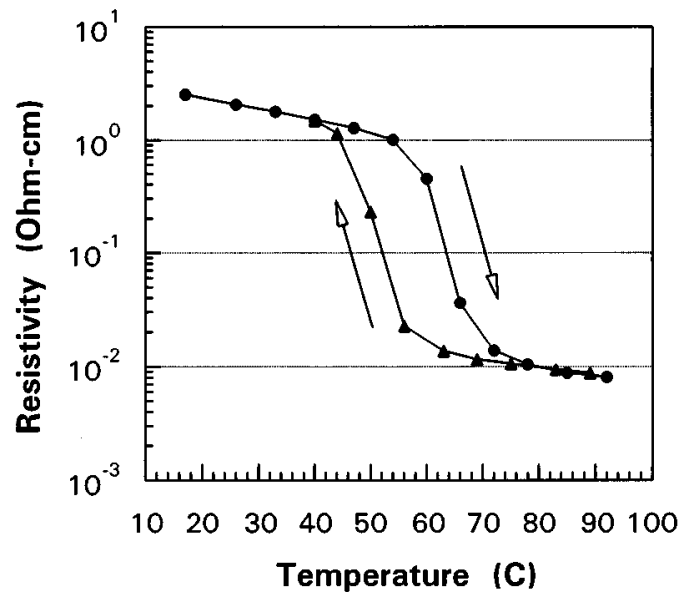

FIG. 1. $\mathrm{VO}_{2}$ film resistivity vs temperature for increasing and decreasing temperature scans.

temperature $<250{ }^{\circ} \mathrm{C}$, followed by annealing in a static nitrogen atmosphere at a temperature $\geqslant 290{ }^{\circ} \mathrm{C}$. The films were verified to be polycrystalline, stoichiometric $\mathrm{VO}_{2}$ by TEM/ TED (transmission electron microscopy/transmission electron diffraction) and x-ray analyses. The electrical resistivity and optical transmission and reflection spectra were identical to those for conventionally processed $\mathrm{VO}_{2}$ films of similar thickness. ${ }^{13}$ The electrical resistivity of the tested film is shown in Fig. 1 as a function of increasing and decreasing temperature. The semiconducting-metallic phase transition and its associated hysteresis are clearly shown. During the transient optical experiments, the film was held just below the phase transition onset temperature, between 39 and $41^{\circ} \mathrm{C}$, by an electrically heated substrate holder with an embedded thermistor. The entire heater and sample assembly was placed in a foam plastic container for thermal isolation. The optical transmission and reflection spectra of the film are shown in Fig. 2 for both the cold (semiconducting) state at $20^{\circ} \mathrm{C}$ and the hot (metallic) state at $90{ }^{\circ} \mathrm{C}$. The Ti:sapphire and $\mathrm{HeNe}$ probe laser wavelengths are indicated on the spectra. The film used in our experiments was deposited on a glass microscope slide and was $32 \mathrm{~nm}$ thick. The film thickness was determined by fitting the measured transmission

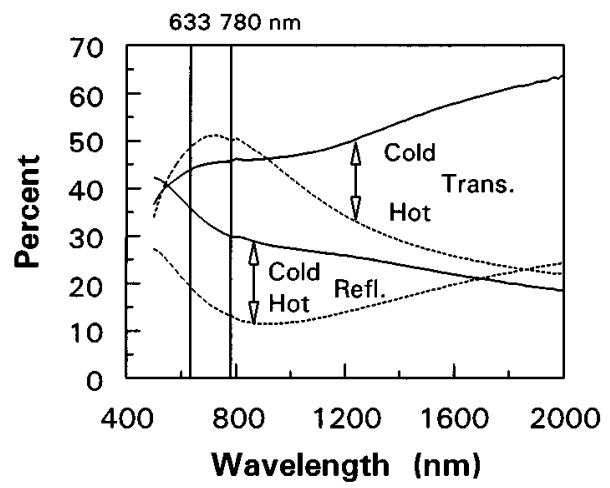

FIG. 2. $\mathrm{VO}_{2}$ film transmission and reflection spectra for the cold (semiconducting) state at $20{ }^{\circ} \mathrm{C}$ and the hot (metallic) state at $90{ }^{\circ} \mathrm{C}$. The vertical lines denote the $\mathrm{cw}$ probe wavelength of $633 \mathrm{~nm}$ and the femtosecond pump-probe wavelength of $780 \mathrm{~nm}$.

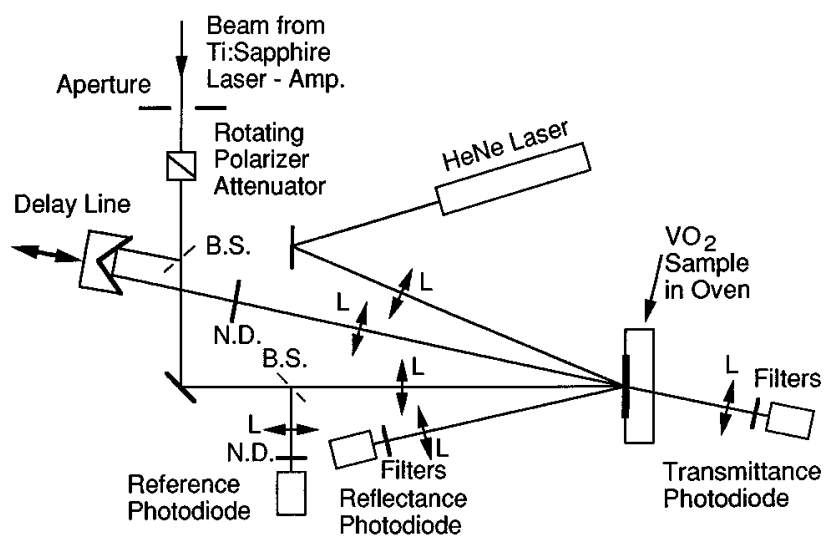

FIG. 3. Experimental setup for measuring the optical dynamics of a $\mathrm{VO}_{2}$ film when excited by a femtosecond Ti:sapphire laser at $780 \mathrm{~nm}$. L is a lens, B.S. is a beam splitter, and N.D. is a neutral density filter.

and reflection versus wavelength (from 500 to $2000 \mathrm{~nm}$ ) of the semiconducting state to that calculated by a thin-film model using reported values for the complex permitivity of a $\mathrm{VO}_{2}$ thin film. ${ }^{14}$

The optical setup used to conduct the pump-probe measurements was relatively conventional,,${ }^{15}$ and the layout is shown in Fig. 3. The laser source was a cw passively modelocked Ti:sapphire laser at $780 \mathrm{~nm}$ wavelength with a regenerative amplifier operating at $20 \mathrm{~Hz}$ and a grating pulse compressor. $^{16,17}$ The pulsewidth was measured by background-free, sum frequency generation in the sample plane and was in the range of 450-550 fs full width at half maximum (FWHM) [assuming a $\operatorname{sech}^{2}$ pulse intensity profile]. The femtosecond probe beam and the cw HeNe beam diameters at the sample plane were adjusted to be less than one-third of the pump beam diameter. Beam overlap and the beam diameters at the sample plane were measured using a charge coupled device (CCD) camera with neutral density filters. The pump beam average power was measured just after the focusing lens, and the power was adjusted to be just sufficient to excite the complete phase transition. This resulted in a pump fluence of $3.7 \mathrm{~mJ} / \mathrm{cm}^{2}$ at the sample plane, in good agreement with the film temperature, heat capacity, and the latent heat of the phase transition.

Experimental transient data for each run was recorded either for transmission or reflection, and the nanosecond transient wave form was recorded using a digitizing oscilloscope and a fast photodiode. For each delay line position, both the desired peak voltage (reflection or transmission) and the peak reference voltage were measured using the digitizing oscilloscope. In addition, frequent measurements were taken with the pump beam blocked and used to correct for small changes as the delay was changed. Data for transmission and reflection were first corrected for the probe pulse behavior with the pump beam blocked and then fit at large negative time delay to the transmission and reflection measured by the spectrometer at $780 \mathrm{~nm}$.

\section{TRANSIENT OPTICAL PARAMETERS}

The resulting data is a record of transient transmission and reflection versus delay time from -7 to $400 \mathrm{ps}$. It was 
(a)

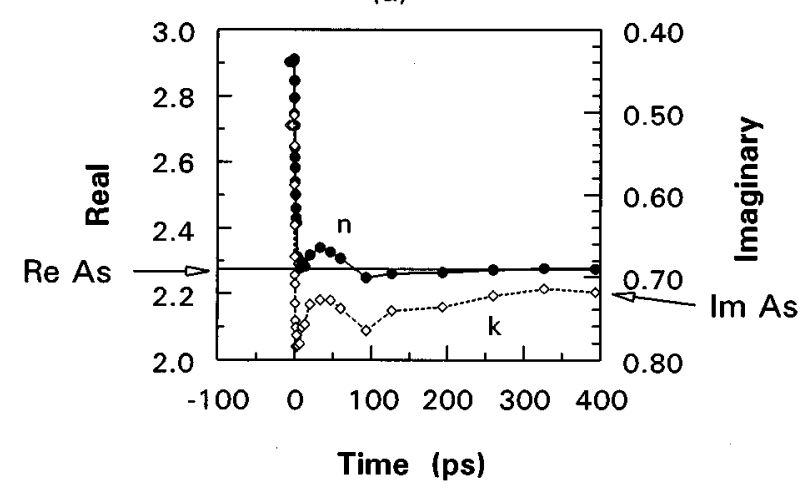

(b)

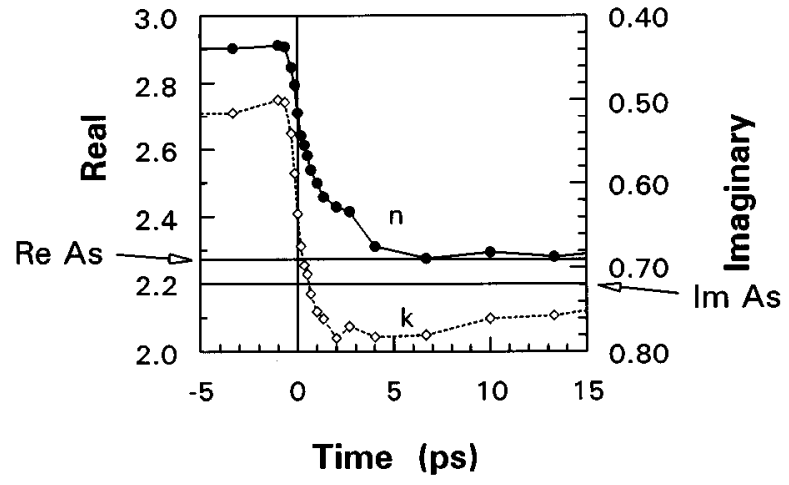

FIG. 4. Complex index of refraction for a $\mathrm{VO}_{2}$ film vs time derived from the measured reflection and transmission data for two time scales: (a) -100 to $400 \mathrm{ps}$ and (b) -5 to $15 \mathrm{ps}$. Note that the imaginary part of the refractive index, $k$, is plotted on the right-hand scale and that the direction of this scale is inverted. Arrows and horizontal lines denote the asymptotic values of $n$ and $k$ (determined at $400 \mathrm{ps}$ ). The laser excitation fluence was $3.7 \mathrm{~mJ} / \mathrm{cm}^{2}$.

immediately apparent that the $\mathrm{VO}_{2}$ film reached an optical state very close to the final state in less than 5 ps. The observed change at $400 \mathrm{ps}$ was equal to the change observed when the sample was heated through the phase transition by static means. This was determined by heating the sample through the phase transition, with and without femtosecond excitation applied, while monitoring the nanosecond and static optical parameters with the cw HeNe laser. Thus, the final reflection and transmission state reached by pulsed laser excitation was identical to that reached by equilibrium heating.

Conversion of the data to a refractive index format was done using a thin-film model for the $\mathrm{VO}_{2}$ film and substrate. A coherent reflection was assumed from the film-substrate interface, and an incoherent reflection was assumed for the substrate rear interface since the 500 fs pulse was shorter than the substrate thickness. A numerical solver routine was used to obtain a value for $n$ and $k$ at each delay time given the measured values of transmission and reflection. The entire data set for complex refractive index, $\tilde{n}=n-j k$, is shown in Fig. 4(a) while the time interval near zero delay is expanded in Fig. 4(b). In the expanded graph, asymptotic values (at $400 \mathrm{ps)}$ ) for both $n$ and $k$ are shown as horizontal lines. Note that the scale for the imaginary index is inverted on both graphs.

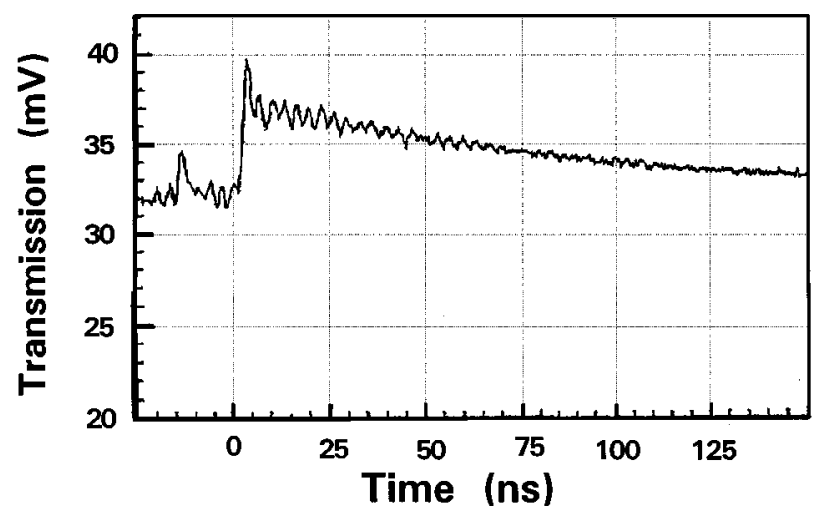

FIG. 5. Measured nanosecond transmission transient for $\mathrm{VO}_{2}$ probed with a $633 \mathrm{~nm} \mathrm{cw}$ HeNe laser.

The real part of the refractive index, $n$, was just over halfway to the metallic value by the end of the $500 \mathrm{fs}$ excitation pulse. It then decreased more slowly to around the steady-state value by $5 \mathrm{ps}$. On the other hand, the imaginary part of the refractive index, $k$, was equal to the metallic value by the end of the excitation laser pulse and overshot by $36 \%$ at 7 ps. After smaller fluctuations, both $n$ and $k$ had reached their final values by $300 \mathrm{ps}$.

For delay times longer than $1 \mathrm{~ns}$, the film behavior was determined by the reflection and transmission of the $\mathrm{cw}$ $\mathrm{HeNe}$ probe laser. If the pump-laser energy was not in excess of that required for the phase transition, the $\sim 100$ ns decay back to the semiconducting state was consistent with heat conduction to the substrate. A typical nanosecond time scale excitation and cooling curve for $\mathrm{VO}_{2}$ transmission is shown in Fig. 5. The pump-laser fluence was $3.7 \mathrm{~mJ} / \mathrm{cm}^{2}$, just sufficient to excite the complete phase transition. If excess excitation (greater than about 1.5 times the energy required for the phase transition) was applied to the sample, additional overshoot and equilibration effects were observed on this time scale, especially in the reflection transient.

\section{ANALYSIS AND PLASMA MODEL}

For the interval from 0.5 to $15 \mathrm{ps}$ in Fig. 4(b), both $n$ and $k$ are greater in magnitude than their corresponding equilibrium metallic values: $n$ lies closer to the semiconducting value, and $k$ has overshot the metallic value. This deviation of both $n$ and $k$ could be explained by the presence of an electron plasma of both higher electron density and higher electron collision frequency than the equilibrium metallicphase plasma. We take the permittivity of $\mathrm{VO}_{2}$ to be of the form of a constant contribution due to lattice vibrations and electronic transitions, plus a contribution due to free carries that is dependent on optical frequency $\omega$. This is written as

$$
\begin{aligned}
\tilde{\boldsymbol{\epsilon}}=\tilde{\boldsymbol{\epsilon}}_{t}+\tilde{\boldsymbol{\epsilon}}_{\mathrm{fc}}= & \tilde{\epsilon}_{t}+\epsilon_{0}\left[\frac{-N e^{2}}{\epsilon_{0} m^{*}\left(\nu^{2}+\omega^{2}\right)}\right. \\
& \left.-j \frac{N e^{2} \nu}{\epsilon_{0} m^{*} \omega\left(\nu^{2}+\omega^{2}\right)}\right],
\end{aligned}
$$

where $\tilde{\epsilon}_{t}$ is the contribution due to phonon and electron transitions, $\tilde{\epsilon}_{\mathrm{fc}}$ is the contribution due to the free carrier plasma, 


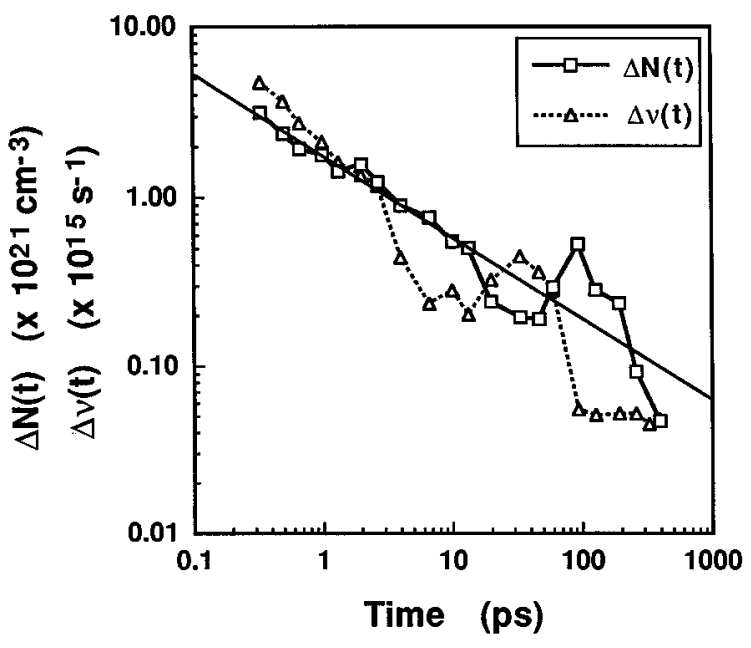

FIG. 6. $\Delta N$ and $\Delta \nu$ vs delay time as derived from modeling the behavior of $n$ and $k$ by a free-electron plasma. A power law dependence described has been fit to the data for $\Delta N$ as shown by the solid straight line.

$N$ is the electron density, $m^{*}$ is the electron effective mass, and $\nu$ is the electron collision frequency (damping or energyloss rate). In the model, $m^{*}$ is assumed to be the freeelectron rest mass, $m_{e}$, since its effect cannot be separated from $N$. Values of $m^{*}$ reported for metallic $\mathrm{VO}_{2}$ are near $m_{e} \cdot{ }^{10,11}$ The phonon and electron transition part of the permittivity, $\tilde{\epsilon}_{t}$, was calculated using the equations and empirically fit parameters of Verleur. ${ }^{14}$ Then the electron plasma parameters $N$ and $\nu$ were adjusted at each value of optical delay such that the computed values for $\tilde{\epsilon}$ (and $\tilde{n}$ ) matched the experimentally derived ones.

It is expected that at $780 \mathrm{~nm}$, the fitted steady-state values for $N$ and $\nu$ (at $400 \mathrm{ps)} \mathrm{should} \mathrm{be} \mathrm{relatively} \mathrm{close} \mathrm{to} \mathrm{those}$ derived in Verleur's model ${ }^{14}$ and those measured by Thomas and Chain ${ }^{18}$ using electron energy-loss spectroscopy (EELS). A small error is expected since our spectroscopic values for $\epsilon^{\prime}$ and $\epsilon^{\prime \prime}$ differ slightly from Verleur's values. In fact, the fitted steady-state values from our data at $780 \mathrm{~nm}$ were $N=6.3 \times 10^{21} \mathrm{~cm}^{-3}$ and $\nu=2.1 \times 10^{15} \mathrm{~s}^{-1}$ in good agreement with Verleur's values of $8.0 \times 10^{21} \mathrm{~cm}^{-3}$ and $\nu=1.0 \times 10^{15}$ $\mathrm{s}^{-1}$ (converted from esu and electron volt units) and Thomas and Chain's values of $N=5.5 \times 10^{21} \mathrm{~cm}^{-3}$ and $\nu=1.4 \times 10^{15}$ $\mathrm{s}^{-1}$.

The fitted plasma parameter data are displayed in terms of deviation from the steady-state values: $\Delta N(t)=N(t)$ $-N_{\text {steady state }}$ and similarly for $\Delta \nu(t)$. The fitted parameters from 300 fs to 400 ps are shown in Fig. 6. Data for delay times less than zero could not be fit with any values of $N$ and $\nu$, and data during the pump-probe overlap time are not reliable because of pulse coupling effects. First, one notices that the decay of both parameters is globally monotonic. Exceptions for $N$ and $\Delta N$ after 60 ps and $\nu$ and $\Delta \nu$ after 20 ps are small and are likely less than the experimental error. Second, both a large percentage increase in electron collision frequency and a proportionally smaller percentage increase in the electron density are required to fit the transient data. Third, the decay cannot be represented by an exponential or sum of exponential terms. However, as plotted in a $\log -\log$ format, the data can be least-squares fit to a power law de- pendence of the form $\Delta N(t)=1.8 \mathrm{t}^{-0.48}$ (shown in Fig. 6) and $\Delta \nu(t)=1.9 \mathrm{t}^{-0.65}$ (not shown in Fig. 6). A time dependence of $1 / \sqrt{t}$ is suggested by this data.

Two possible physical interpretations are suggested by this time dependence for $\Delta N$ and $\Delta \nu$. The first is that a $1 / \sqrt{t}$ dependence is observed as the time dependent part of many diffusion problems. This suggests that diffusion of either carriers or a related hidden variable might play a role in the equilibration of the metallic phase of $\mathrm{VO}_{2}$. The electron collision frequency is presumably connected to the same process. The second possibility is that $\Delta N(t)=N(1) t^{-1 / 2}$ is an explicit solution to the differential Eq.

$$
\frac{d \Delta N(t)}{d t}=-\frac{1}{2 N^{2}(1)}[\Delta N(t)]^{3} .
$$

This kind of decay can have a physical significance; it is the dependence observed for decay dominated by a three-body collision process. For comparison, a single body process leads to the familiar exponential decay and a decay rate proportional to the concentration of the decaying specie. A twobody relaxation process, such as Auger recombination, has a decay rate proportional to the product of two specie concentrations. And likewise, the decay rate for a three-body decay is proportional to a triple product of concentrations. For each additional body in the collision process, the collision rate is multiplied by the probability of finding another particle simultaneously present in the collision interaction volume, Probability $=(\Delta N)$ (Interaction Volume), which is expected to be a small number. We consider that the probability is very low for decay arising from a three-body collision of excess carriers to completely dominate one- and two-body decay processes. Thus, we select diffusion as the more likely explanation for the observed behavior.

\section{CONCLUSIONS}

It appears that a metallic plasma model for $\mathrm{VO}_{2}$ can successfully fit the transient behavior of the optical parameters after the femtosecond optical excitation pulse. The model fit implies a small $(<20 \%)$ increase in the electron density, along with a larger $(>200 \%)$ increase in the electron collision frequency at the end of the excitation laser pulse $(t=0.5 \mathrm{ps})$. The decay of both of these parameters back to their equilibrium values of $N=6.3 \times 10^{21} \mathrm{~cm}^{-3}$ and $\nu=2.1 \times 10^{15} \mathrm{~s}^{-1}$ follows the same, nearly monotonic time dependence. Thus, the decay of these two plasma parameters is connected to the same physical mechanism (an apparent variable such as excess carrier density or perhaps a hidden variable).

Fitting the time dependence of $\Delta N$ and $\Delta \nu$ yielded a $1 / \sqrt{t}$ dependence and two possible interpretations, but we believe a diffusion process to be the more probable of the two. The involvement of a diffusion process in the equilibration of metallic $\mathrm{VO}_{2}$ is also physically plausible since diffusion from micro-inhomogeneities in the phase transition is possible. Although no anomalous optical scattering from such inhomogeneities was observed on passing through the laser excited phase transition, this only rules out inhomogeneities larger than the optical wavelength. Two articles sug- 
gest inhomogeneities in the phase transition and have calculated inhomogeneity sizes less than the optical wavelength. ${ }^{19,20}$

Additional spectrally resolved, femtosecond pump-probe experiments in which the probe light is a wide bandwidth continuum might give further information about the optically excited phase transition. Given time-resolved spectra in the near-IR, visible, and near-UV regions, a better fit to an appropriate oscillator model should better identify the state reached in the first $0.5 \mathrm{ps}$ and the equilibration mechanism that leads to the nonexponential decay of the plasma density and collision rate.

We conclude that, if indeed the $\mathrm{VO}_{2}$ state after end of the optical excitation pulse can be represented by its metallic phase plus a higher density, more highly damped electron plasma, then the transition to the metallic phase must have occurred during the interval of the pump pulse. This then establishes an upper limit of roughly $400 \mathrm{fs}$ for the switching time of the phase transition.

\section{ACKNOWLEDGMENTS}

M. F. Becker acknowledges partial support from the University Research Institute at The University of Texas and from the University of Paris 11, Faculty of Physics. The $\mathrm{VO}_{2}$ samples were fabricated under the sponsorship of the Energy Research in Applications Program of the Texas Higher Education Coordinating Board.

${ }^{1}$ D. P. Kien, J. C. Loulergue, and J. Etchepare, in Ultrafast Phenomena VIII, edited by J.-L. Martin, A. Migus, G. A. Mourou, and A. H. Zewail (Springer, New York, 1993), p. 368.

${ }^{2}$ T. P. Dougherty, G. P. Wiederrecht, K. A. Nelson, M. H. Garrett, H. P. Jensen, and C. Warde, Science 258, 770 (1992).
${ }^{3}$ D. Hulin, A. Mysyrowicz, L. L. Chase, A. Antonetti, J. Etchepare, G. Grillon, and A. Migus, in Ultrafast Phenomena IV, edited by D. H. Austin and K. B. Eisenthal (Springer, New York, 1984), p. 126.

${ }^{4}$ W. R. Roach and I. Balberg, Solid State Comm. 9, 551 (1971).

${ }^{5}$ M. F. Becker, R. M. Walser, and R. W. Gunn, in Picosecond Phenomena, edited by C. V. Shank, E. P. Ippen, and S. L. Shapiro (Springer, New York, 1978), p. 236.

${ }^{6}$ R. M. Walser and M. F. Becker, in Laser-Solid Interactions and Laser Processing-1978 MRS Conference, edited by S. D. Ferris, H. J. Leamy, and J. M. Poate (AIP, New York, 1979), p. 117.

${ }^{7}$ A. A. Bugaev, V. V. Gudyalis, B. P. Zakharchenya, and F. A. Chudnovskii, JETP Lett. 34, 430 (1982).

${ }^{8}$ A. A. Bugaev, V. V. Gudyalis, and A. V. Klochkov, Sov. Phys. Solid State 26, 887 (1984).

${ }^{9}$ M. F. Becker, A. B. Buckman, R. M. Walser, T. Lépine, P. Georges, and A. Brun, Appl. Phys. Lett. 65, 1507 (1994).

${ }^{10}$ A. S. Barker, Jr., H. W. Verleur, and H. J. Guggenheim, Phys. Rev. Lett. 17, 1286 (1966).

${ }^{11}$ C. N. Berglund and H. J. Guggenheim, Phys. Rev. 185, 1022 (1969).

${ }^{12}$ R. M. Walser, H. C. Joung, M. F. Becker, R. W. Bené, and A. B. Buckman, SPIE Symposium on Smart Structures and Materials, Albuquerque, N. M., 1993 (unpublished).

${ }^{13}$ S. M. Babulanam, T. S. Eriksson, G. A. Niklasson, and C. G. Granqvist, Proc. SPIE 692, 8 (1986).

${ }^{14}$ H. W. Verleur, A. S. Barker, Jr., and C. N. Berglund, Phys. Rev. 172, 788 (1968). This reference contains data for $\epsilon^{\prime}$ and $\epsilon^{\prime \prime}$ vs wavelength based on measured reflection and transmission for a $100 \mathrm{~nm}$ thick $\mathrm{VO}_{2}$ thin film. Fitted parameters are given for an electron oscillator model for $\epsilon^{\prime}$ and $\epsilon^{\prime \prime}$.

${ }^{15}$ M. F. Becker, A. B. Buckman, R. M. Walser, T. Lépine, P. Georges, and A. Brun, Proc. SPIE 2189, 400 (1994).

${ }^{16}$ J. Squier, F. Salin, and G. Mourou, Opt. Lett. 16, 324 (1991).

${ }^{17}$ D. Strickland and G. Mourou, Opt. Commun. 56, 219 (1985).

${ }^{18}$ M. Thomas and E. E. Chain, Thin Solid Films 204, L1 (1991).

${ }^{19}$ Y. M. Gerbshtein, T. V. Smirnova, and F. A. Chudnovskii, JETP Lett. 25, 445 (1977).

${ }^{20}$ V. I. Emel'yanov and A. L. Semenov, Sov. Phys. Solid State 32, 1790 (1990). 